\title{
Influence of scintillation on quality of ozone monitoring by GOMOS
}

\author{
V. F. Sofieva ${ }^{1}$, V. Kan $^{2}$, F. Dalaudier ${ }^{3}$, E. Kyrölä ${ }^{1}$, J. Tamminen ${ }^{1}$, J.-L. Bertaux ${ }^{3}$, A. Hauchecorne ${ }^{3}$, D. Fussen , and $^{4}$ \\ F. Vanhellemont ${ }^{4}$ \\ ${ }^{1}$ Earth observation, Finnish Meteorological Institute, Helsinki, Finland \\ ${ }^{2}$ Organization of Russian Academy of Sciences A.M. Obukhov Institute of Atmospheric Physics RAS, Moscow, Russia \\ ${ }^{3}$ LATMOS, Université Versailles Saint-Quentin; CNRS/INSU, Verrières-le-Buisson, France \\ ${ }^{4}$ Institut d'Aeronomie Spatiale de Belgique, Brussels, Belgium
}

Received: 6 May 2009 - Published in Atmos. Chem. Phys. Discuss.: 29 May 2009

Revised: 30 October 2009 - Accepted: 16 November 2009 - Published: 7 December 2009

\begin{abstract}
Stellar light passing through the Earth atmosphere is affected by refractive effects, which should be taken into account in retrievals from stellar occultation measurements. Scintillation caused by air density irregularities is a nuisance for retrievals of atmospheric composition. In this paper, we consider the influence of scintillation on stellar occultation measurements and on the quality of ozone retrievals from these measurements, based on experience of the GOMOS (Global Ozone Monitoring by Occultation of Stars) instrument on board the Envisat satellite.

In GOMOS retrievals, the scintillation effect is corrected using scintillation measurements by the fast photometer. We present quantitative estimates of the current scintillation correction quality and of the impact of scintillation on ozone retrievals by GOMOS. The analysis has shown that the present scintillation correction efficiently removes the distortion of transmission spectra caused by scintillations, which are generated by anisotropic irregularities of air density. The impact of errors of dilution and anisotropic scintillation correction on the quality of ozone retrievals is negligible. However, the current scintillation correction is not able to remove the wavelength-dependent distortion of transmission spectra caused by isotropic scintillations, which can be present in off-orbital-plane occultations. This distortion may result in ozone retrieval errors of $0.5-1.5 \%$ at altitudes $20-40 \mathrm{~km}$. This contribution constitutes a significant percentage of the total error for bright stars. The advanced inversion methods that can minimize the influence of scintillation correction error are also discussed.
\end{abstract}

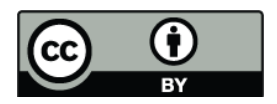

Correspondence to: V. F. Sofieva (viktoria.sofieva@fmi.fi)

\section{Introduction}

The stellar occultation measurements have a set of beneficial features that are important for long-term monitoring, such as a self-calibration measurement principle, global coverage, good vertical resolution, and a wide altitude range of measurements from the troposphere to the thermosphere. However, using stars imposes certain requirements on the instrument and retrievals. Since stars are point sources of quite low-intensity light, special instruments are needed for recording stellar spectra. The stellar spectra observed through the Earth atmosphere are not only attenuated by absorption and scattering (this phenomenon is used in reconstruction of chemical composition of the atmosphere), but they are also modified by refractive effects.

A nearly exponential decrease of the atmospheric air density with altitude is responsible for a refractivity gradient that leads to the bending of rays coming from a star (the lower the ray perigee (tangent) altitude, the larger the bending). Refraction in the atmosphere transforms parallel incident rays into diverging beams, thus resulting in dilution of the light intensity registered at the satellite level. This effect is known as refractive attenuation (or refractive dilution). The dependence of atmospheric refractivity on wavelength leads to a differential bending of rays of different color in the atmosphere; this effect is known as chromatic refraction.

The chromatic refraction and refractive dilution are related to a "smooth" dependence of refractive index on wavelength and altitude. However, the air density and, as a consequence, atmospheric refractivity, always has fluctuations caused by the atmospheric processes such as internal gravity waves (IGW), turbulence, different kinds of atmospheric instabilities. The interaction of light waves with refractivity irregularities results in scintillation, i.e., fluctuations in the measured

Published by Copernicus Publications on behalf of the European Geosciences Union. 
intensity of stellar light. If the stellar light passed through the atmosphere is recorded at a satellite with a high-frequency device, the measured intensity fluctuations may exceed the mean value by several hundred percent. The scintillations that are generated by random irregularities of air density do not change the mean intensity of the measured stellar light.

It is known that there are two types of air density irregularities in the Earth stratosphere: anisotropic irregularities, which are stretched along the Earth surface (generated by mainly by internal gravity waves), and isotropic irregularities (turbulence), which appear as a result of gravity wave breaking and due to different instabilities. These irregularities produce scintillations at the observation plane. Hereafter, we will refer to the scintillations generated by anisotropic and isotropic air density irregularities as to anisotropic and isotropic scintillations, for short.

Scintillations do not produce any bias in the statistics of an ensemble of reconstructed profiles because of their random nature. They only result in fluctuations in retrieved profiles of atmospheric constituents. The influence of scintillation on the quality of ozone reconstruction in the stellar occultation experiment has been discussed by Polyakov et al. (2001). The authors have considered ozone retrievals from stellar occultation measurements in the wavelength range 580-900 nm (272 spectral channels, MSX/UVISI spectrometer). They estimated the influence of scintillation on quality of ozone monitoring via numerical simulation of scintillations and subsequent error propagation. The authors concluded that stellar scintillations may result in noticeable reduction (at least, by a few percent) in accuracy of ozone reconstruction, if the scintillation effect is not corrected.

The GOMOS (Global Ozone Monitoring by Occultation of Stars) instrument on board the Envisat satellite (sun synchronous orbit at the altitude $\sim 800 \mathrm{~km}$, launched in March 2002) includes UV-VIS-IR spectrometers for monitoring of ozone and other trace gases in the atmosphere. The GOMOS spectrometers record stellar spectra transmitted through the atmosphere continuously with a sampling frequency of $2 \mathrm{~Hz}$ as the occulted star sets behind the Earth limb (for illustration of the measurement principle, see Fig. 1 in Kyrölä et al., 2004). In addition, GOMOS is equipped with two fast photometers sampling synchronously the stellar flux in lowabsorption wavelength regions, blue $(473-527 \mathrm{~nm})$ and red $(646-698 \mathrm{~nm})$, at a sampling frequency of $1 \mathrm{kHz}$. The photometers and the spectrometers have the same field of view (Kyrölä et al., 2004, Fig. 2). The stellar scintillation measurements by the fast photometers are used for temperature profiling with a high vertical resolution (Dalaudier et al., 2006), for studying small-scale processes in the stratosphere (Gurvich et al., 2005; Sofieva et al., 2007a, b, 2009; Gurvich et al., 2007), and for the correction of scintillations in the spectrometer data. The scintillation correction applied in the GOMOS inversion aims at reduction of rms fluctuations in retrieved profiles due to scintillation.
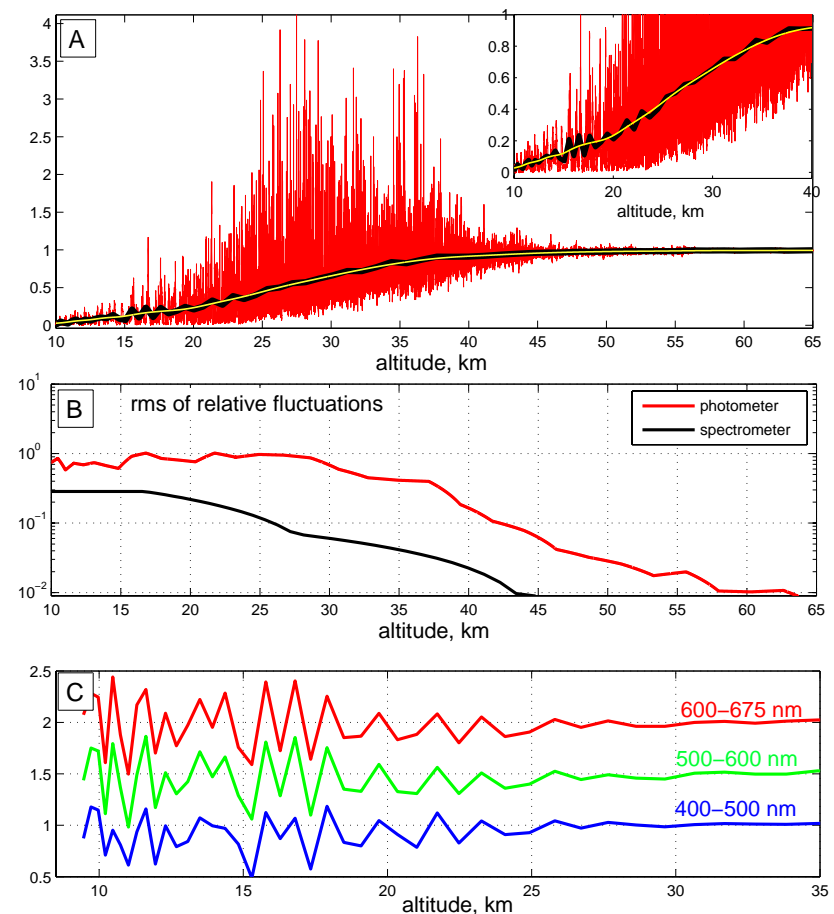

Fig. 1. (A): Scintillation measurements by the GOMOS red photometer in the occultation of Sirius R02833/S001 (18 September 2002, $37 \mathrm{~S}, 164 \mathrm{E}$ ); the scintillation averaged to $2 \mathrm{~Hz}$ (black line), and the smooth signal $\langle I\rangle$ obtained from the scintillation data by using filtering with the Hanning window having the cut-off scale $3 \mathrm{~km}$ (yellow line). (B): rms of relative fluctuations of intensity $\frac{I-\langle I\rangle}{\langle I\rangle}$, for the GOMOS red photometer and the spectrometer (The data of Fig. 1a are used. For computing rms, $3 \mathrm{~km}$ samples with $50 \%$ overlapping are used for the photometer signal and $6 \mathrm{~km} \mathrm{sam}$ ples with $66 \%(2 / 3)$ overlapping are used for the spectrometer signals.). (C): Fluctuations caused by scintillation in GOMOS transmittances before the scintillation correction, for the considered occultation R02883/S001. The color lines corresponding to the mean transmittance in the pointed wavelength regions are offset by 0.5 in $\mathrm{y}$-axis for a better visibility.

In this paper, we give the description of the scintillation correction that is applied in GOMOS processing and discuss its quality and limitations. In our analyses, we combine theoretical estimates, experimental results and simulation. We restrict the scope of this work to consider the influence of scintillation only on ozone retrievals, as it is the main target of the GOMOS mission. However, other retrieved constituents are affected in a similar way.

The paper is organized as follows. Section 2 is dedicated to the description of the GOMOS scintillation correction. The quality of this scintillation correction is discussed in Sects. 3 and 4. Section 5 is dedicated to quantitative estimates of ozone retrieval errors caused by incomplete scintillation correction. A discussion of inversion methods that allow minimizing the influence of scintillation on accuracy of retrievals and a summary conclude the paper. 

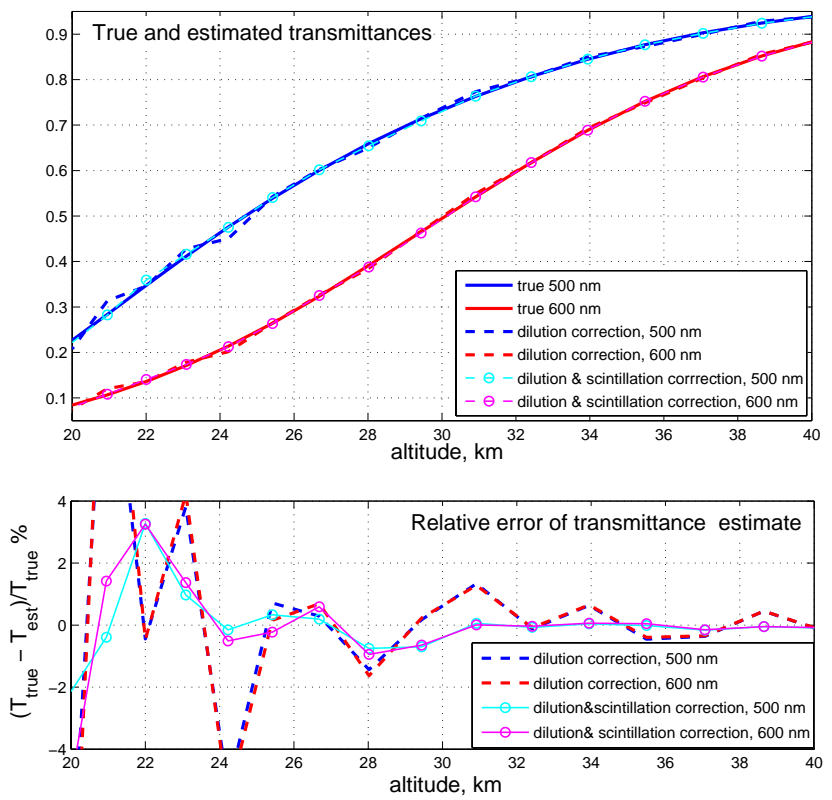

Fig. 2. Top: true (solid lines) and estimated transmittances due to absorption and scattering. Bottom: relative error of the transmittance estimates, with and without scintillation correction.

\section{The GOMOS scintillation correction}

Let us illustrate the magnitude of fluctuations in stellar spectra that are caused by scintillations. Figure 1a shows an example of scintillation measurements by the GOMOS fast photometer (red line). The rms of relative fluctuations of intensity recorded by the photometer rapidly grows with decreasing altitude until it saturates at values $\sim 1$ below $30 \mathrm{~km}$ (Fig. 1b). In Fig. 1 and all subsequent figures, altitudes characterizing the GOMOS measurements and retrievals correspond to the tangent altitudes. Ozone and other trace gases are retrieved from GOMOS UV-VIS spectrometer measurements, which have a significantly lower sampling rate than photometers, $2 \mathrm{~Hz}$. However, the photometer signal averaged down to $2 \mathrm{~Hz}$ still exhibits fluctuations (Fig. 1a, black line), thus showing the possible modulation of the spectrometer signals caused mainly by scintillation. The depth of this modulation (shown also in Fig. 1b) ranges from $\sim 4 \%$ in case of vertical (in orbital plane) occultations up to $\sim 20 \%$ in case of strongly oblique (off orbital plane) occultations. The fluctuations caused by scintillations are well observed in the transmittances plotted as a function of altitude (Fig. 1c); they are often well correlated for different wavelengths. The amplitude of fluctuations in spectrometer signals caused by scintillation exceeds the instrumental noise, especially for very bright stars. The "scintillation noise" is a nuisance for ozone retrieval by influencing the final error budget, and it should be corrected as much as possible before starting the inversion procedure.
The GOMOS processing starts with the computation of atmospheric transmission spectra $T_{\mathrm{atm}}$, which are obtained by dividing the spectra measured at different tangent altitudes by the reference spectrum, measured above the atmosphere. These transmission spectra contain spectral signatures of absorption and scattering in the atmosphere, which are also modified by refractive effects. Since absorption and refraction affect the atmospheric transmission spectra $T_{\text {atm }}$ independently, we can write it as a product (GOMOS ESL, 2007):

$T_{\text {atm }}(\lambda, t)=T_{\text {ext }}(\lambda, t) T_{\text {ref }}(\lambda, t)$

where $T_{\text {ext }}$ is the transmittance due to absorption and scattering, and $T_{\text {ref }}$ represents the combined effect of refraction and scintillation.

In the GOMOS retrieval, the component due to refractive effects and scintillation $T_{\text {ref }}$ is estimated and eliminated from the atmospheric transmission data. The refractive term, $T_{\text {ref }}$, is presented in the form

$T_{\text {ref }}(\lambda, t)=T_{d}(\lambda, t) T_{s c}(t)$,

where the component $T_{d}$ corresponding to regular refractive effects (refractive dilution) is modulated by the scintillation component $T_{s c}$. In the limit of weak refraction regime (non-crossing rays), the dilution term $T_{d}$ can be estimated as (Dalaudier et al., 2001)

$\hat{T}_{d}(\lambda)=\frac{1}{1+L \frac{d \alpha_{\mathrm{ref}}(\lambda, p)}{d p}}$,

where $L$ is a distance from the tangent point to the satellite, $p$ is an impact parameter and $\alpha_{\text {ref }}$ is a refractive angle (hereafter, variables with "hats" are used for denoting "estimates"). In GOMOS processing, the refractive angle $\alpha_{\text {ref }}$ is estimated from ray tracing through the combined ECMWF and MSIS90 (Hedin, 1991) air density field.

The idea of the GOMOS scintillation correction is described in (Dalaudier et al., 2001). For the scintillation correction, measurements of the red photometer are used, as they have a better signal-to-noise ratio. The estimation of scintillation modulation $T_{s c}$, consists of detecting fluctuations from the scintillation measurements. It is determined as relative fluctuations of the photometer signal:

$\hat{T}_{s c}(t)=\frac{I(t)}{\langle I\rangle}$,

where $I(t)$ is the photometer signal and $\langle I\rangle$ is the smoothed photometer signal. The Hanning filter (e.g., Oppenheim and Schafer, 1989) of variable width, with FWHM corresponding to $\approx 3 \mathrm{~km}$ movement of the tangent point, is used for the smoothing. The estimate of the refractive component, $\hat{T}_{\text {ref }}$, corresponding to each tangent altitude $h$ and to each wavelength of the spectrometer measurements, is obtained then by 
averaging $\hat{T}_{d}(\lambda, t) \hat{T}_{s c}(t)$ over the $\Delta t=0.5 \mathrm{~s}$ integration time of spectrometers:

$\hat{T}_{\text {ref }}(\lambda, h)=\frac{1}{\Delta t} \int_{\Delta t} \hat{T}_{d}(\lambda, t) \hat{T}_{s c}(t) d t$.

Finally, the measured transmission spectra are divided by the estimated refraction component, thus giving the transmission due to absorption and scattering $T_{\mathrm{ext}}$ :

$\hat{T}_{\text {ext }}(\lambda, h)=\frac{T_{\text {atm }}(\lambda, h)}{\hat{T}_{\text {ref }}(\lambda, h)}$.

The transmission spectra $\hat{T}_{\text {ext }}(\lambda, h)(6)$ provide the basis for retrievals of the atmospheric constituent profiles in the GOMOS data processing.

Due to chromatic shift, spectrometer measurements in each channel correspond to their own tangent altitude. Using high-vertical-resolution fast photometer measurements and the known wavelength dependence of refractivity allows correction of scintillations, which takes the chromatic shift into account, for each spectrometer channel. Since the photometer wavelengths are located in the low-absorption region, it is assumed that fluctuations due to extinction are much smaller than scintillations.

In the GOMOS scintillation correction, it is assumed that light rays of different color pass through the same air density vertical structures, thus the signal perturbations at different wavelengths are identical after appropriate shifting and stretching resulting from the chromatic refraction effect. This hypothesis is always satisfied in vertical occultations and it is true for scintillations generated by anisotropic irregularities, practically for all obliquities. However, this hypothesis may be violated in oblique occultations if isotropic turbulence is well developed. Validity of these assumptions is discussed in (Dalaudier et al., 2001; Kan et al., 2001), and will be considered further in Sect. 4 of this paper.

\section{Quality of anisotropic scintillation correction}

Under the assumption of strong anisotropy of air density irregularities and provided the mean refraction is perfectly known and scintillations are weak, we can expect that the scintillation correction described above eliminates almost perfectly the scintillation-dilution component from the measured transmission spectra. The main error of the anisotropic scintillation correction comes from impossibility of complete separating the dilution and scintillation terms. Other error sources are noise in the photometer data and the fact that the photometer records not a monochromatic intensity but the averaged intensity over the wavelength band of the optical filter. At altitudes below $25-30 \mathrm{~km}$, the weak scintillation assumption is violated due to multi-path propagation and ray crossing, thus resulting in further degradation of the scintillation correction accuracy.

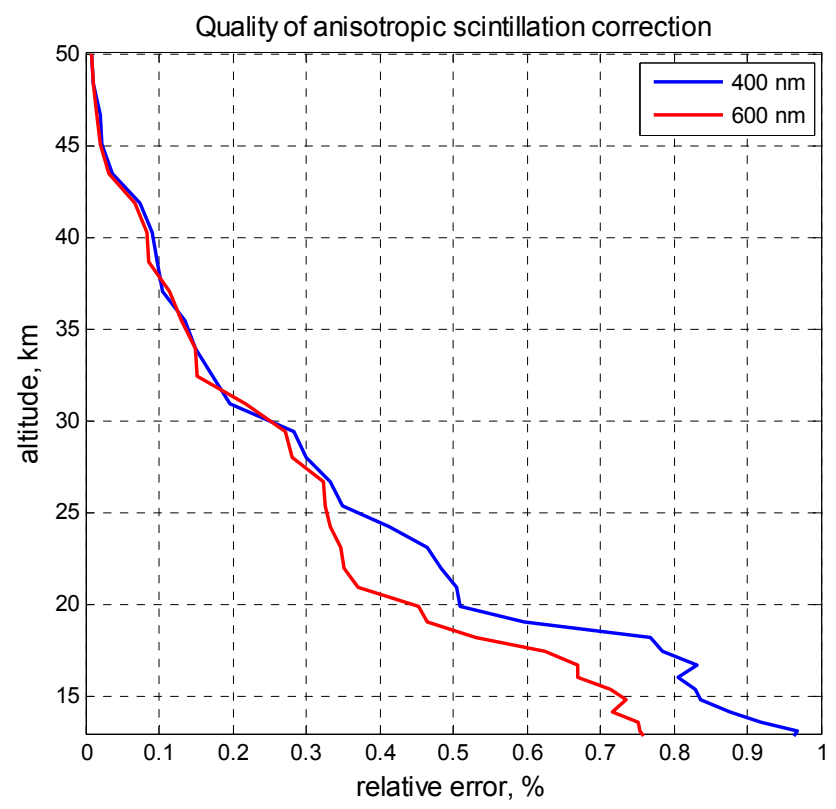

Fig. 3. Relative error in estimated transmittances caused by error of anisotropic scintillation 3 correction: results of Monte Carlo simulations.

In order to estimate the best quality of the scintillation correction, the noise-free signal of the red photometer (anisotropic scintillations) was simulated with the scintillation model. Details of scintillation simulations are given in (Dalaudier and Sofieva, 2009). The transmission due to absorption and scattering was simulated with LIMBO (Kyrölä et al., 1999). For simulation of the atmospheric transmission $T_{\mathrm{atm}}(\lambda, t)$ measured by the GOMOS spectrometer, we used the following approach. First, monochromatic scintillations at $1 \mathrm{kHz}$ sampling frequency corresponding to the wavelengths of each pixel were simulated (with the extinction effect included), and then the signal was integrated down to $2 \mathrm{~Hz}$ sampling frequency of the spectrometer.

Figure 2 illustrates the quality and usefulness of the GOMOS scintillation correction: most of the modulation caused by scintillation, which is well observed in the curves corresponding to the dilution correction only, is eliminated by the applied scintillation correction. The rms of the residual (non-corrected) scintillation is below $1 \%$ for altitudes above $\sim 20 \mathrm{~km}$ altitude range.

To estimate average quality of the anisotropic scintillation correction, we carried out Monte Carlo simulations (100 runs) of the scintillation correction described above, with different scintillation realizations. The relative error of the anisotropic scintillation correction (i.e., the error in estimated transmittances) is shown in Fig. 3. It demonstrates that the GOMOS scintillation correction efficiently eliminates modulation caused by anisotropic scintillations: the remaining error is below $1 \%$. 


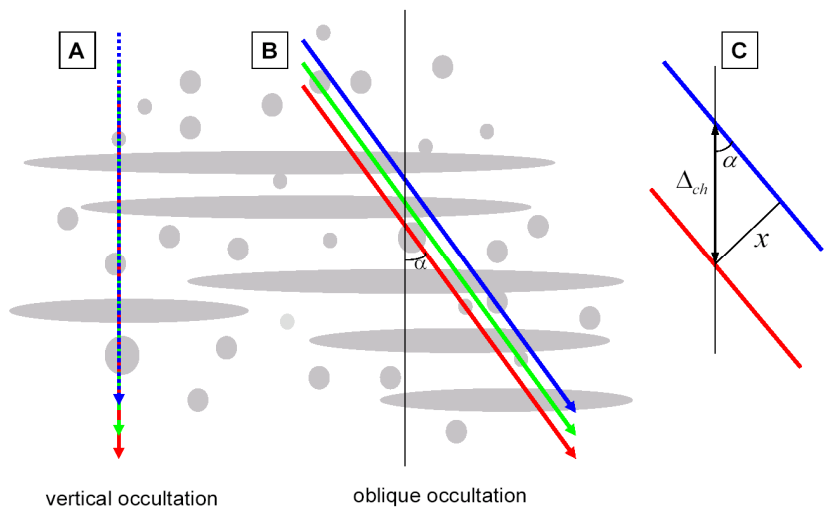

Fig. 4. Colored lines: trajectories of the intersection points of light rays and the phase screen (i.e., the plane perpendicular to the light rays and passed through the tangent point, which produces the same phase modulation of propagated light waves as the extended atmosphere; for details of the phase screen approximation, see e.g. (Ishimaru, 1978; Dalaudier et al., 2001; Gurvich and Brekhovskikh, 2001) in vertical (A) and oblique (B) occultations. Grey ovals schematically show anisotropic irregularities of air density and grey circles denote isotropic irregularities. (C): parameters ralated to chromatic separation of rays: $\Delta_{c h}$ is the vertical chromatic shift, $\alpha$ is obliquity of the occultation, $x=\Delta_{c h} \sin \alpha$ is the distance between colored rays.

The residual error of correction of anisotropic scintillation, as well as the dilution correction error $\delta T_{\text {ref }}$, leads to perturbation of the estimated transmittances due to absorption and scattering:

$\hat{T}_{\mathrm{ext}}=\frac{T_{\mathrm{ext}} T_{\mathrm{ref}}}{\hat{T}_{\mathrm{ref}}}=\frac{T_{\mathrm{ext}} T_{\mathrm{ref}}}{T_{\text {ref }}\left(1+\frac{\delta T_{\mathrm{ref}}}{T_{\text {ref }}}\right)} \approx T_{\mathrm{ext}}\left(1-\frac{\delta T_{\text {ref }}}{T_{\text {ref }}}\right)$

Since the dependence of $\delta T_{\text {ref }} / T_{\text {ref }}$ on wavelength is negligibly small in the case of anisotropic scintillations (Kan et al., 2001), the erroneous correction of dilution and anisotropic scintillation does not modify the spectral shape of the transmittance due to absorption and scattering, but changes only its value (equivalently, the optical depth values are shifted by a constant). Such modulation of the transmittance spectra leads to coincident fluctuations in the retrieved profiles of horizontal column densities. The simulations have shown that the sensitivity of ozone retrieval to the spectrally flat perturbation of transmittance spectra is negligible, $2 \%$ transmittance perturbation results in only $0.001 \%$ perturbation in ozone horizontal column density. The sensitivity of other constituents $\left(\mathrm{NO}_{2}, \mathrm{NO}_{3}\right.$, aerosols) having lower optical depth is significantly larger than that of ozone; these effects will be considered in future publications.

\section{Impact of isotropic scintillations}

\subsection{Qualitative explanation}

In the presence of isotropic small-scale air density irregularities, the main assumption of the GOMOS scintillation correction - that light rays of different color pass through the same refractivity structures - can be violated (Fig. 4). In reality, we always observe a mixture of anisotropic and isotropic scintillations. In vertical occultations, the colored rays pass through the same refractivity structures (Fig. 4a), thus both isotropic and anisotropic scintillations are correlated for different wavelengths. In oblique occultations, the anisotropic scintillations are still well correlated (Fig. 4b). The correlation of isotropic scintillation depends on chromatic separation of ray trajectories corresponding to different wavelengths $\lambda_{1}$ and $\lambda_{2}$. If the separation of ray trajectories (Fig. 4c) becomes larger than $\sim \max \left(l_{K}, \rho_{F}\right)$, the isotropic scintillations become uncorrelated (Kan, 2004; Gurvich et al., 2005). Here $\rho_{F}$ is the Fresnel scale $\left(\rho_{F} \approx\left(\frac{\sqrt{\lambda_{1} \lambda_{2}} L}{2 \pi}\right)^{1 / 2} \sim 0.45-0.6 \mathrm{~m}\right.$ for GOMOS) and $l_{K}$ is the Kolmogorov's scale (e.g., Gurvich and Kan, 2003a), which is typically $\sim 0.2-0.3 \mathrm{~m}$ in the stratosphere (Gurvich and Kan, 2003b).

Obviously, the GOMOS scintillation correction is able to remove only perfectly correlated fluctuations. Furthermore, applying this correction to isotropic scintillations, as if they were anisotropic, introduces an additional error.

\subsection{Characterization of the scintillation correction er- ror}

The residuals $R(\lambda)=T_{\text {ext }}(\lambda)-T_{\text {mod }}(\lambda)$, i.e., the difference between measured and modeled transmittances, can be used as an indicator of the inversion quality. If the model describes perfectly measurements and provided instrumental noise is non-correlated, the residuals will be close to white noise. This is the case for nearly vertical occultations (Fig. 5, bottom). In oblique occultations, residuals display wavelengthcorrelated oscillating features, which are observed clearly in the case of bright stars (Fig. 5, top). In the case of dim or medium-brightness stars (having visual magnitude larger than $\sim 2$ ), such oscillations are not observed because of significant noise background. The residual oscillations are the structures that are not corrected and not explained by the model. The amplitude of residual oscillations is maximal in the altitude range $\sim 20-40 \mathrm{~km}$. The residual fluctuation structures are evidently correlated in wavelength, and the correlation length increases with wavelength at a given altitude and increases with altitude for a given wavelength range.

These features, which are observed even in individual cases (like in Fig. 5, top) were confirmed by the statistical correlation analysis of residuals, which was performed for different altitudes, obliquity angles and wavelength ranges. The auto-correlation function (ACF) of residual fluctuations, being presented as a function of wavelength, has a clear 

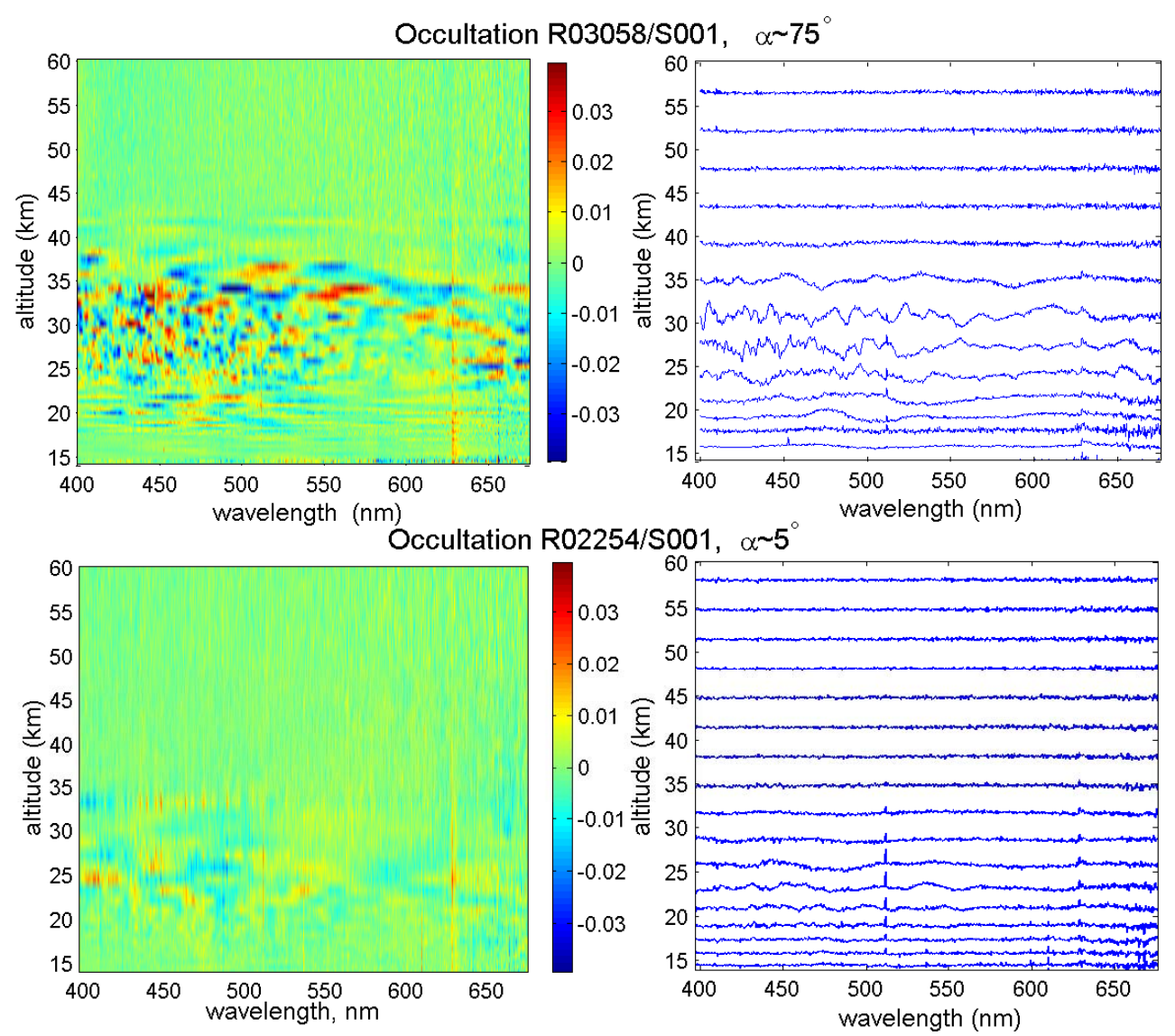

Fig. 5. Top: residuals in the oblique occultation of Sirius R03058/S001 $\left(\alpha \sim 75^{\circ}\right)$. Bottom: residuals in close to vertical occultation of Sirius R02254/S001. Left: color plot; right: scaled residuals (by the factor 50) at selected altitudes.

dependence on altitude (the higher altitude, the wider $\mathrm{ACF}$ ), on obliquity (the smaller obliquity, the wider ACF, for the same altitude), and on wavelength (the decay of ACF is more rapid for blue wavelengths compared to red ones). However, being presented as a function of the chromatic distance between the colored rays $x=\Delta_{c h} \sin \alpha$, where $\Delta_{c h}$ is the vertical chromatic shift, and $\alpha$ is the obliquity of the occultation (Fig. 4c), ACFs of residuals become very close to each other. This is illustrated in Fig. 6, which shows the experimental autocorrelation function of residual fluctuations presented as a function of the vertical chromatic shift $\Delta_{c h}$ and as a function of chromatic separation $x=\Delta_{c h} \sin \alpha$. This is in good agreement with the theory of isotropic scintillations generated by locally isotropic turbulence, which predicts the correlation of isotropic scintillations at two wavelengths depending on chromatic separation of colored ray trajectories. When the distance between trajectories of colored rays $x=\Delta_{c h} \sin \alpha$ exceeds $\sim \max \left(l_{K}, \rho_{F}\right)$, correlation of bichromatic isotropic scintillations rapidly drops. At the same time, the performed correlation analysis of GOMOS residual fluctuations has supported the hypotheses that the "oscillations" in residuals are caused by isotropic scintillations.
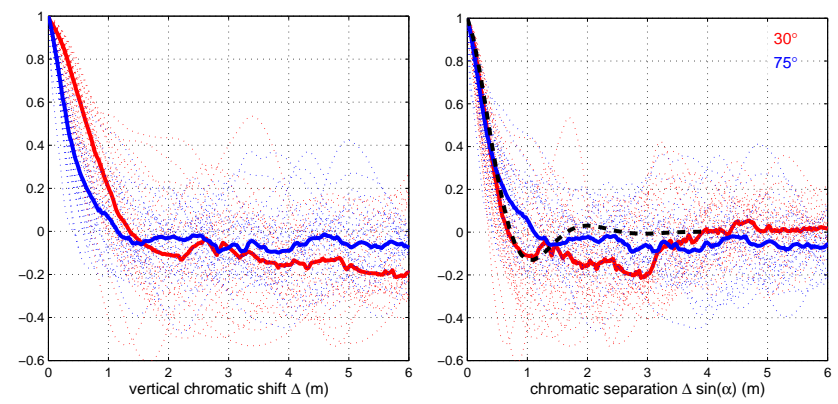

Fig. 6. ACF of residual fluctuations as a function of vertical chromatic shift (left) and as a function of chromatic distance $x$ (right), for two series of sequential occultations of Sirius: with obliquities $\sim 75^{\circ}$ and $\sim 30^{\circ}$. The altitude is $30 \mathrm{~km}$. Bold lines: median. The dashed bold line in the right subplot indicates the parameterization given by Eq. (10).

Assuming that (i) the dilution estimate is error-free, (ii) the anisotropic component of the scintillation is estimated with the error $\delta T_{s c}^{a n}: \hat{T}_{s c}^{a n}=T_{s c}^{a n}\left(1+\frac{\delta T_{s c}^{a n}}{T_{s c}^{a n}}\right)$, and (iii) the modulation due to isotropic scintillation can be presented in the 
form $T_{s c}^{i s}=1+\frac{\delta I^{i s}}{I^{i s}}$, then the estimated transmittance due to absorption and scattering can be approximated as:

$\hat{T}_{\mathrm{ext}}=\frac{T_{\mathrm{atm}}}{\hat{T}_{d} \hat{T}_{s c}^{a n}} \approx$

$\frac{T_{\mathrm{ext}} T_{d} T_{s c}^{a n} T_{s c}^{i s}}{T_{d} T_{s c}^{a n}\left(1+\frac{\delta T_{s c}^{a n}}{T_{s c}^{a n}}\right)} \approx T_{\mathrm{ext}}+T_{\mathrm{ext}}\left(\frac{\delta I^{i s}}{I^{i s}}-\frac{\delta T_{s c}^{a n}}{T_{s c}^{a n}}\right)$.

The factorization of the transmission due to scintillation $T_{s c} \approx T_{s c}^{a n} T_{s c}^{i s}$ in (8) approximates the assumption that anisotropic and isotropic irregularities generate statistically independent fluctuations in measured intensity. This is valid for weak scintillations. The additional term, $\varepsilon_{s c}=T_{\operatorname{ext}}\left(\frac{\delta I^{i s}}{I^{i s}}-\frac{\delta T_{s c}^{a n}}{T_{s c}^{a n n}}\right)$, represents the scintillation correction error that exists in GOMOS measurements in case of oblique occultations in turbulent atmosphere. It is assumed to be a Gaussian random variable with zero mean and covariance matrix $\mathbf{C}_{s c}$ :

$\mathbf{C}_{s c}=\left\{c_{i j}\right\}, c_{i j}=\sigma_{i} \sigma_{j} B_{i j}$,

where indices $i$ and $j$ denote spectrometer pixels corresponding to wavelengths $\lambda_{i}$ and $\lambda_{j}$, and $\sigma$ is the amplitude and $B$ is the correlation function of off-diagonal elements. The theoretical estimates of cross-correlation of isotropic scintillation for spectrometer channels can be used for defining the correlation function $B$ of the scintillation modelling error. They can be computed using Eqs. (A7) and (A8) from (Kan et al., 2001), or approximated by

$B\left(\lambda_{i}, \lambda_{j}\right)=B(\xi)=\exp \left(-0.4|\xi|^{1.15}\right) J_{0}(1.5 \xi)$,

where $\xi$ is the ratio of the chromatic separation of rays corresponding to wavelengths $\lambda_{i}$ and $\lambda_{j}$ to the Fresnel scale $\rho_{F}$

$\xi=\frac{\Delta_{c h}\left(\lambda_{i}, \lambda_{j}\right) \sin \alpha}{\rho_{F}}$,

and $J_{0}$ is the Bessel function of zero order. We found that the correlation function of residuals is narrower at upper altitudes (above $\sim 45 \mathrm{~km}$ ) than that predicted by (10). Most probably, this is caused by the applied scintillation correction. To take this effect into account, the parameter $\xi$ in (10) should be replaced by $\xi / s$, where an empirically derived expression for $s$ is proposed: $s=1-\exp \left(-\left(\xi_{0} / 5\right)^{2}\right), \xi_{0}=\xi(375$ $\mathrm{nm}, 425 \mathrm{~nm})$. An example of the correlation function of the spectrometer pixels $B\left(\lambda_{i}, \lambda_{j}\right)$ at $30 \mathrm{~km}$ is shown in Fig. 7; it is also indicated in Fig. 6 (right).

For the amplitude of the scintillation correction error, the following approximation is proposed:

$\sigma(z, \lambda, \alpha)=T_{\text {ext }} \sigma_{\text {iso }}(z, \lambda, \alpha) \sqrt{\left(1-b_{p h \_p} B(\lambda, \lambda \text { red })\right)}$

where $\sigma_{\text {iso }}(z, \lambda, \alpha)$ is the rms of isotropic scintillations (relative fluctuations of intensity) in spectrometer channels, and the term $1-b_{p h \_s p} B\left(\lambda, \lambda_{\text {red }}\right)$ takes into account the influence of the scintillation correction procedure.

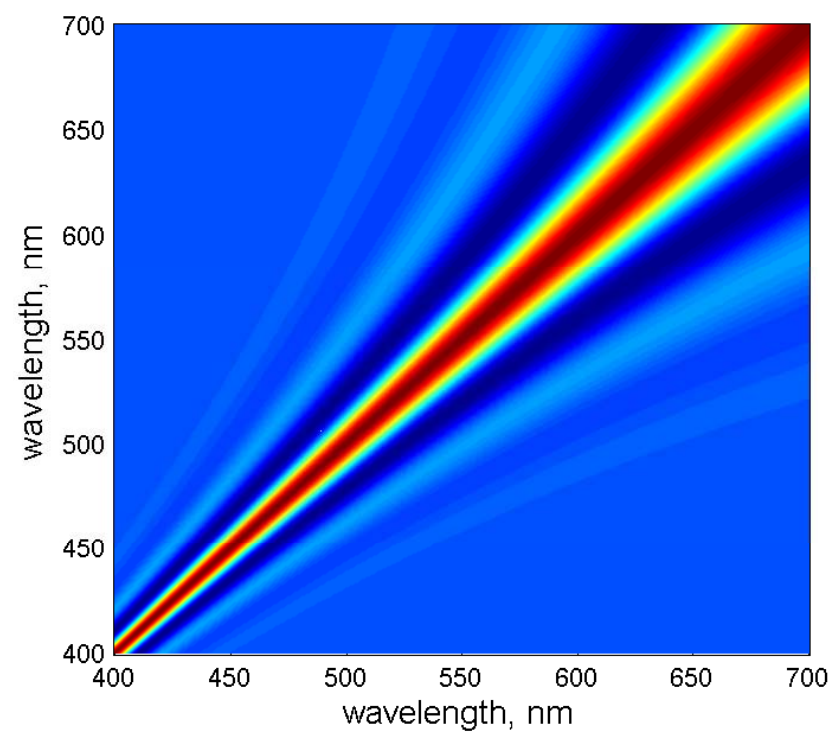

Fig. 7. Cross-correlation function (Eq. 10) of the spectrometer pixels at $30 \mathrm{~km}$, obliquity $\sim 30^{\circ}$

In Eq. (12), $\sigma_{\text {iso }}(z, \lambda, \alpha)$ is parameterized as:

$\sigma_{\text {iso }}(z, \lambda, \alpha)=\sigma_{0}(z) \frac{\rho(z)}{\rho_{0}(z)} \sqrt{\frac{v_{0}}{v(\alpha)}}\left(\frac{\lambda}{\lambda_{\text {red }}}\right)^{-1 / 3}$

Here $\sigma_{0}(z)$ is the "standard" profile of isotropic scintillation variance in the spectrometer channels, which was estimated using red photometer data $\left(\lambda_{\text {red }}=672 \mathrm{~nm}\right)$ from all occultations of Canopus in 2003 with obliquity $\alpha \sim 50^{\circ}$ by the method explained in (Sofieva et al., 2007a), and $\rho_{0}(z)$ is the average air density profile in the considered data set. The factors in Eq. (13) give the dependence of $\sigma_{\text {iso }}$ on wavelength $\lambda$, obliquity $\alpha$ (via dependence of full ray velocity $v$ in the phase screen on $\alpha$ ), and the mean air density $\rho(z)$.

In (12), $b_{p h_{-} p}$ is the ratio of isotropic scintillation variances of smoothed red photometer and spectrometer signals for $\lambda_{\text {red }}=672 \mathrm{~nm}$, which is parameterized as:

$b_{p h \_s p}=\exp \left(-0.105\left(\frac{\Delta_{c h}^{p h} \sin \alpha}{\rho_{F}}\right)^{1.5}\right)$,

where $\Delta_{c h}^{p h}$ is the vertical chromatic shift for wavelengths $672 \pm 25 \mathrm{~nm}$, corresponding to the width of the red photometer optical filter. $B\left(\lambda, \lambda_{\text {red }}\right)$ is the correlation coefficient between the smoothed red photometer and spectrometer channels, which is defined in the same way as the correlation of spectrometer channels, Eq. (10).

Figure 8 shows the experimental estimates of the amplitude of scintillation correction error, which was computed as $\sqrt{\sigma_{R}^{2}-\sigma_{\varepsilon}^{2}}$, i.e., the difference between the observed variance of residual fluctuations $\sigma_{R}^{2}$ and the predicted noise variance $\sigma_{\varepsilon}^{2}$, from the set of sequential occultations of Sirius 

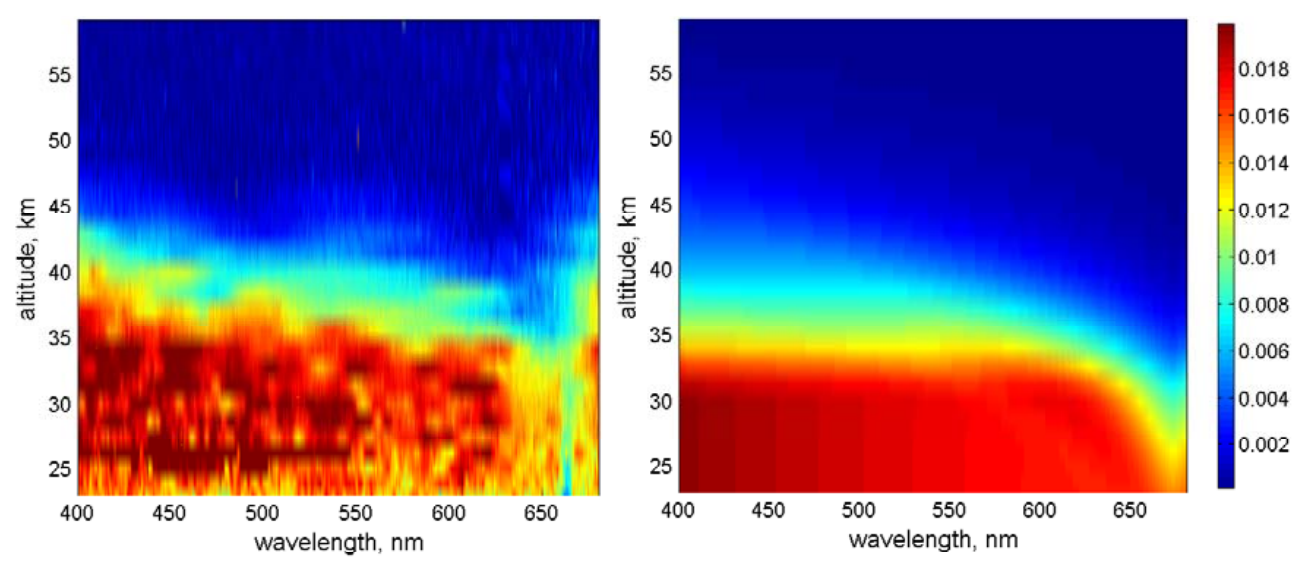

Fig. 8. Left: experimental estimates of the amplitude of scintillation correction error. Right: parameterization of scintillation error amplitude, for the same obliquity.

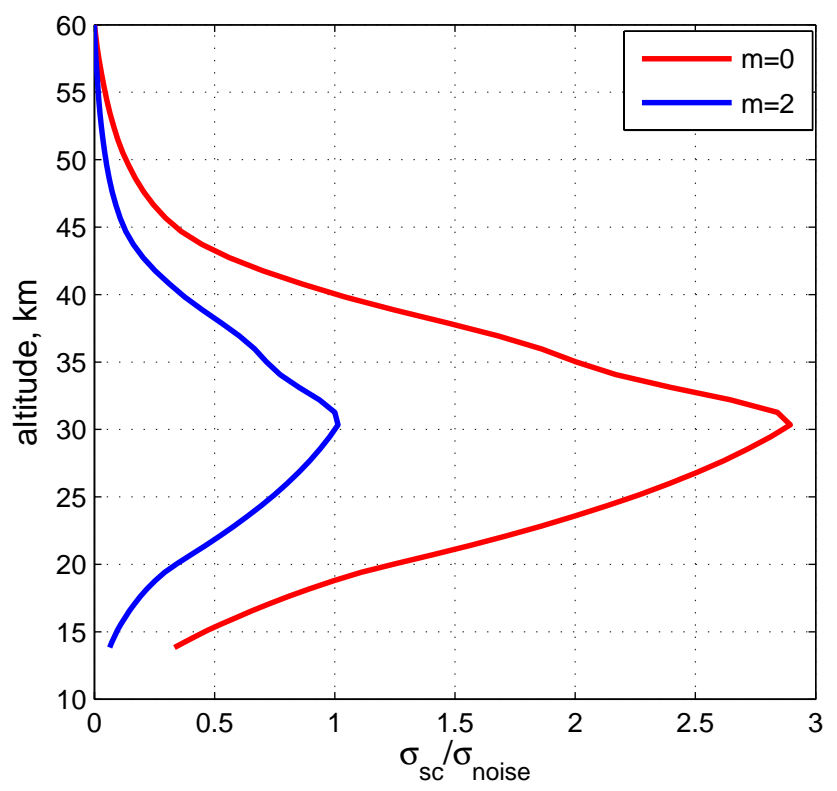

Fig. 9. Altitude dependence of ratio $r=\frac{\sigma_{s c}}{\sigma_{\text {noise }}}$ at $\lambda=500 \mathrm{~nm}$, for stars of magnitude 0 and 2 and effective temperature $T=11000 \mathrm{~K}$. The obliquity of the occultation is $\alpha=30^{\circ}$.

in January 2005 (obliquity $\sim 45^{\circ}$ ), and the parameterization of scintillation error given by Eqs. (12)-(14), for the same obliquity. The experimental estimates are in good agreement with the proposed parameterization (Fig. 8, right). As observed in Fig. 8, isotropic scintillations are corrected only in a very narrow wavelength band close to the central wavelength of the red photometer.

The covariance matrix of the transmission errors, $\mathbf{C}_{\text {tot }}$, can be presented as a sum of two matrices (provided errors are Gaussian):

$\mathbf{C}_{\text {tot }}=\mathbf{C}_{\text {noise }}+\mathbf{C}_{s c}$, where the diagonal matrix $\mathbf{C}_{\text {noise }}$ corresponds to the measurement noise, while the non-diagonal matrix $\mathbf{C}_{s c}$ corresponds to the scintillation correction error. The altitude dependence of the ratio of scintillation and noise standard deviations $r=\frac{\sigma_{s c}}{\sigma_{\text {noise }}}$ is shown in Fig. 9, for the wavelength $500 \mathrm{~nm}$. For very bright stars, the isotropic scintillation correction error can be more than twice as large as the instrumental noise.

\section{Propagation of the scintillation correction error in the GOMOS inversion}

In the GOMOS data processing, the inversion is split into two parts: the spectral inversion and the vertical inversion (Kyrölä et al., 1993). The spectral inversion problem can be written in the form:

$\hat{\boldsymbol{T}}_{\text {ext }}=\exp (-\boldsymbol{\Sigma} \boldsymbol{N})+\boldsymbol{\varepsilon}_{\text {tot }}$

where $\hat{\boldsymbol{T}}_{\text {ext }}$ are measured transmittances after the dilutionscintillation correction, $\boldsymbol{\Sigma}$ is the matrix of effective crosssections, $\boldsymbol{N}$ are horizontal column densities and $\boldsymbol{\varepsilon}_{\text {tot }}$ represents the error term (noise and modeling errors). In the spectral inversion, horizontal column densities are retrieved from the atmospheric transmission data $\hat{T}_{\text {ext }}(\lambda, h)$, for each tangent altitude. In the vertical inversion, vertical profiles are reconstructed from the collection of horizontal column densities.

In the GOMOS inversion, errors of horizontal column density reconstruction are given by the Levenberg-Marquardt algorithm used for solving the non-linear spectral inversion problem (16). The statistical error of the retrieved parameters is characterized by its covariance matrix, which is computed as Gaussian error propagation using the covariance matrix of measurement noise and the Jacobian matrix provided by the Levenberg-Marquardt algorithm. However, since the degree of non-linearity of the GOMOS spectral inversion problem is not high when transmittances are not too small (Tamminen, 2004), the error of horizontal column density reconstruction 

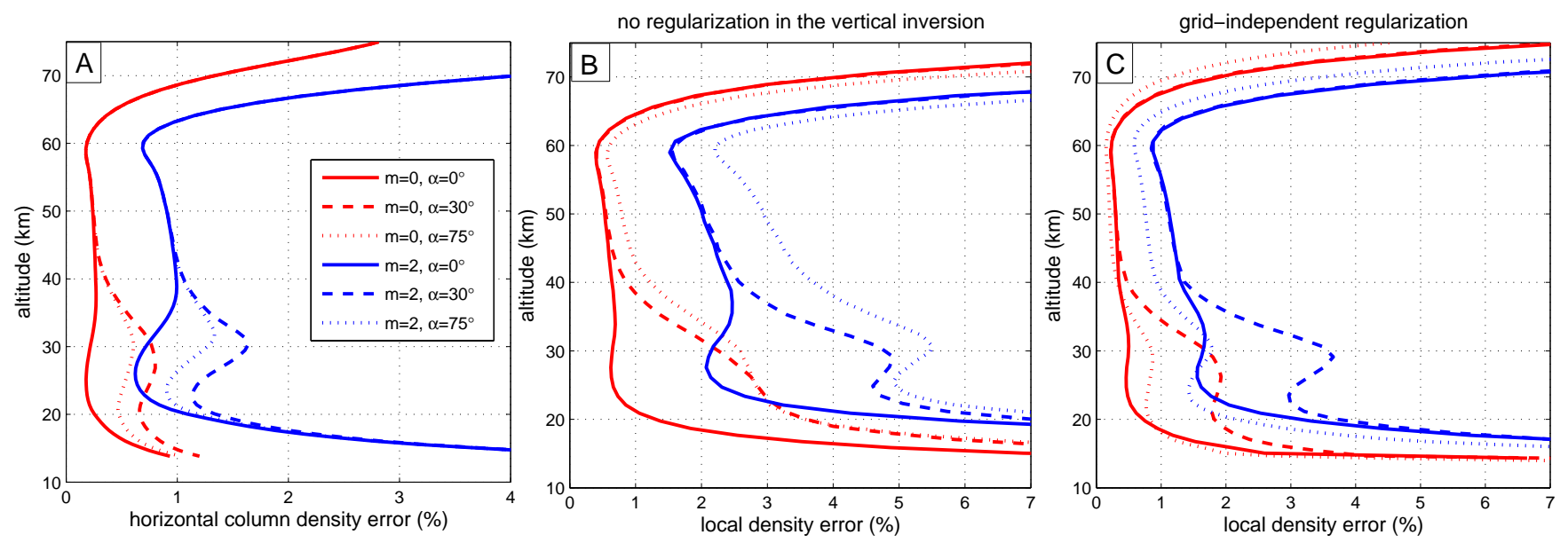

Fig. 10. (a): relative error of the ozone horizontal column density retrievals in vertical $\left(\alpha=0^{\circ}\right)$ and oblique $\left(\alpha \neq 0^{\circ}\right)$ occultations. Error due to non-corrected isotropic scintillations is taken into account. (B): Ozone local density errors; the vertical inversion has been performed without regularization. Vertical sampling resolution is different in vertical and oblique occultations, and this is taken into account. (C): as $\mathrm{B}$, but the grid-independent regularization is applied in the vertical inversion (the vertical resolution of the retrieved profiles is the same for vertical and oblique occultations). The analysis is performed for stars of magnitudes $m=0$ and $m=2$ and of effective temperature $11000 \mathrm{~K}$. Line notations are specified in the legend.

can be estimated via Gaussian error propagation in the linearized spectral inversion:

$\tau=-\ln \left(\hat{\boldsymbol{T}}_{\text {ext }}\right)=\boldsymbol{\Sigma} \boldsymbol{N}+\tilde{\boldsymbol{\varepsilon}}_{\text {tot }}$,

where $\tilde{\varepsilon}_{\text {tot }}=\varepsilon_{\text {tot }} / T_{\text {ext }}$ is the error in the linearized spectral inversion (17). Then the covariance matrix of horizontal column density errors $\mathbf{C}_{N}$ can be obtained as

$\mathbf{C}_{N}=\left(\boldsymbol{\Sigma}^{T} \tilde{\mathbf{C}}_{\text {tot }}^{-1} \boldsymbol{\Sigma}\right)^{-1}$,

where $\tilde{\mathbf{C}}_{\text {tot }}$ is the covariance matrix of the total error $\tilde{\varepsilon}_{\text {tot }}$.

The impact of the non-corrected isotropic scintillations on the ozone retrievals quality is illustrated in Fig. 10. Panel A compares the horizontal column density error estimates for perfect scintillation correction (corresponding to vertical occultations, $\alpha=0^{\circ}$ ) with the error estimates (18) (corresponding to non-corrected isotropic scintillations in oblique occultations). The "turbulence error" results in additional error of $0.5-1 \%$ in horizontal column density reconstruction. Note that the error in significantly oblique occultations is smaller than in moderately oblique occultations (compare lines for $30^{\circ}$ and $75^{\circ}$ obliquity in Fig. 10a). This is due to dependence of isotropic scintillation variance on obliquity (Eq. 13). Although the absolute value of the scintillation correction error is relatively small, the isotropic scintillation constitutes a significant percentage of the total error budget in case of bright stars (because measurement noise is low for bright stars).

The spectral inversion is followed by the vertical inversion aimed at reconstruction of local densities of ozone, $\mathrm{NO}_{2}$, $\mathrm{NO}_{3}$ and aerosols (Kyrölä et al., 2009; Sofieva et al., 2004). The Tikhonov-type regularization is applied in the vertical inversion for its stabilization. It is formulated in the gridindependent way (Tamminen et al., 2004; Sofieva et al.,
2004) so that the actual (target) resolution of the retrieved profiles, which takes into account the smoothing properties by inversion, is independent of the retrieval grid. The regularization parameter depends on the vertical sampling resolution, which can be significantly better in oblique occultations. As a result, more smoothing is applied in oblique occultations, which are affected by isotropic scintillations. Note that an excessive smoothing is prevented by the applied "target resolution" regularization method.

Figure 10b shows the errors of ozone local density for oblique and vertical occultations provided the vertical inversion is performed without regularization. If the regularization is not applied, the incomplete scintillation correction would result in $\sim 1-2 \%$ error in ozone local density retrieval at altitudes $20-40 \mathrm{~km}$. If the grid-independent regularization is applied (Fig. 10c), the scintillation correction error is still visible in oblique occultations of very bright stars at $\sim 20$ $40 \mathrm{~km}$, but it is $\sim 0.5 \%$ for $\alpha=75^{\circ}$ and $\sim 1-1.5 \%$ for $\alpha=30^{\circ}$ versus $\sim 2 \%$ if the regularization is not applied. For typical stars (lines corresponding to visual magnitude $2, \mathrm{~m}=2$ in Fig. 10c) and significantly oblique occultations, the accuracy of ozone local density retrievals can be very similar in oblique and vertical occultations (compare blue solid and dotted lines in Fig. 10c). Since the sampling vertical resolution is twice better for $\alpha=75^{\circ}$ than in vertical occultations $\left(\alpha=0^{\circ}\right)$, the grid-independent regularization applies more smoothing in the oblique occultation than in the vertical one, thus reducing fluctuations in the retrieved profiles caused by both measurement noise and the scintillation correction error. Note that local density errors are smaller in oblique occultations at all altitudes outside the most disturbed altitude region $\sim 20-40 \mathrm{~km}$, for the same reason. 
These estimates of the impact of isotropic scintillation on quality of ozone retrieval were obtained with the aid of the GOMOS data analysis, and therefore they are close to reality. Figure 10a and c shows typical values of ozone retrieval errors induced by the incomplete scintillation correction. The parameterization of the scintillation correction error that uses the chromatic separation of rays enables quantitative characterization of the scintillation correction error for different obliquities of occultations. The obtained parameterization of the scintillation correction (modeling) error can be directly used in the inversion. When the GOMOS inversion is performed using $\mathbf{C}_{\text {tot }}$, the normalized $\chi^{2}$ statistics is close to unity. This indicates that error estimates are close to reality. However, the non-diagonal covariance matrix of the modelling errors reduces the numerical efficiency of the GOMOS spectral inversion. The description of the implementation and assessment of this method will be the subject of future publications.

\section{Summary}

We have presented quantitative estimates of the current scintillation correction quality and of the impact of scintillation on ozone retrievals by GOMOS. The following main conclusions can be drawn from this study:

1. The present scintillation correction efficiently removes the modulation of transmittance spectra caused by anisotropic scintillations.

2. The impact of errors of dilution and anisotropic scintillation correction on quality of ozone retrievals is negligible.

3. The current scintillation can only remove correlated fluctuations in transmission spectra. The modulation of transmission spectra caused by uncorrected isotropic scintillations may result in error of ozone horizontal column density retrievals of $0.5-1 \%$ at altitudes $20-40 \mathrm{~km}$. This contribution to the error budget is significant for bright stars.

4. The grid-independent regularization of Tikhonov type ("target resolution" method) implemented in the GOMOS vertical inversion significantly reduces the retrieval error. By applying more smoothing in oblique occultations, which are affected by incomplete scintillation correction, it makes the retrieval accuracy in oblique occultations less than 1-1.5\% worse than in vertical occultations of the same star. In case of significantly oblique occultations and not very bright stars, the accuracy of ozone retrieval is very similar in the oblique and vertical occultations at altitudes $20-40 \mathrm{~km}$.
Acknowledgements. The authors thank ESA, ACRI-ST and the GOMOS team for the GOMOS data. The authors sincerely thank A. S. Gurvich for insightful discussions and comments related to this paper. The work of V. F. Sofieva was supported by the Academy of Finland (postdoctoral researcher project). The work of V. Kan was supported by RFBR grant 09-05-00180.

Edited by: M. Van Roozendael

\section{References}

Dalaudier F., Kan, V., and Gurvich, A. S.: Chromatic refraction with global ozone monitoring by occultation of stars. I. Description and scintillation correction, Appl. Opt., 40, 866-877, 2001.

Dalaudier, F., Sofieva, V., Hauchecorne, A., Kyrölä, E., Blanot, L., Guirlet, M., Retscher, C., and Zehner, C.: High-resolution density and temperature profiling in the stratosphere using bichromatic scintillation measurements by GOMOS, Proceedings of the First Atmospheric Science Conference, European Space Agency, ISBN: 92-9092-939-1-ISSN 1609-042X, 2006.

Dalaudier, F. and Sofieva, V. F.: Simulation of optical scintillations, GOMOS special issue, Atmos. Chem. Phys., in preparation, 2009.

GOMOS ESL, Algorithm Theoretical Basis Document, version 2.0, http://envisat.esa.int/instruments/gomos/atbd/, 2007.

Gurvich, A. S. and Brekhovskikh, V. L.: Study of the turbulence and inner waves in the stratosphere based on the observations of stellar scintillations from space: a model of scintillation spectra, Waves in Random Media, 11, 163-181, 2001.

Gurvich, A. S. and Kan, V.: Structure of Air Density Irregularities in the Stratosphere from Spacecraft Observations of Stellar Scintillation, 1. Three-Dimensional Spectrum Model and Recovery of Its Parameters, Izvestia, Atmos. Ocean. Phys., 39, 300-310, 2003a.

Gurvich, A. S., and Kan, V.: Structure of Air Density Irregularities in the Stratosphere from Spacecraft Observations of Stellar Scintillation, 2. Characteristic Scales, Structure Characteristics, and Kinetic Energy Dissipation, Izvestia, Atmos. Ocean. Phys., 39, 311-321, 2003b.

Gurvich, A. S., Dalaudier, F., and Sofieva, V. F.: Study of stratospheric air density irregularities based on two-wavelength observation of stellar scintillation by Global Ozone Monitoring by Occultation of Stars (GOMOS) on Envisat, J. Geophys. Res., 110, D11110, doi:10.1029/2004JD005536, 2005.

Gurvich, A. S., Sofieva, V. F., and Dalaudier, F.: Global distribution of $\mathrm{C}_{T}^{2}$ at altitudes $30-50 \mathrm{~km}$ from space-borne observations of stellar scintillation, Geophys. Res. Lett., 34, L24813, doi:10.1029/2007GL031134, 2007.

Hedin, A. E.: Extension of the MSIS thermospheric model into the middle and lower atmosphere, J. Geophys. Res., 96, 1159-1172, 1991.

Ishimaru, A.: Wave propagation and scattering in random media. V.2. Multiple scattering, turbulence, rough surfaces and remote sensing, Academic Press, 572 pp., 1978.

Kan V., Dalaudier, F., and Gurvich, A. S.: Chromatic refraction with global ozone monitoring by occultation of stars. II. Statistical properties of scintillations, Appl. Opt., 40, 878-889, 2001.

Kan, V.: Coherence and correlation of chromatic stellar scintillations in a space-borne occultation experiment, Izvestia, Atmos. 
Ocean. Opt., 17, 725-735, 2004.

Kyrölä, E., Sihvola, E., Kotivuori, Y., Tikka, M., Tuomi, T., and Haario, H.: Inverse theory for occultation measurements: 1. Spectral inversion, J. Geophys. Res., 98, 7367-7381, 1993.

Kyrölä, E., Tamminen, J., Oikarinen, L., Sihvola, E., Verronen, P., and Leppelmeier, G. W.: LIMBO- Limb and occultation measurement simulator, in ESAMS99, European Symposium on Atmospheric Measurements from Space, vol.WPP-161, ESA, Noordwijk, 487-493, 1999.

Kyrölä E., Tamminen J., Leppelmeier G.W., Sofieva V., Hassinen S., Bertaux J.L., Hauchecorne A., Dalaudier F., Cot C., Korablev O., Fanton d'Andon O., Barrot G., Mangin A., Theodore B., Guirlet M., Etanchaud F., Snoeij P., Koopman R., Saavedra L., Fraisse R., Fussen D. and F. Vanhellemont. GOMOS on Envisat: An overview, Adv. Space Res., 33, 1020-1028, doi:10.1016/S0273-1177(03)00590-8, 2004.

Kyrölä, E., Tamminen, J., Sofieva, V. F., et al.: GOMOS retrieval algorithms, GOMOS special issue, in preparation, 2009.

Oppenheim, A. V. and Schafer, R. W.: Discrete-Time Signal Processing, Prentice-Hall, Upper Saddle River, N. J., 1989.

Polyakov, A. V., Timofeev, Yu. M., Gurvich, A. S., Vorobyev, V. V., Kan, V., and Yee, J.-H.: Effect of Stellar Scintillations on the Errors in Measuring the Ozone Content of the Atmosphere, Izv., Atmos. Ocean. Phys. (Engl. transl.), 37(1), 51-60, 2001.
Sofieva, V. F., Tamminen, J., Haario, H., Kyrölä, E., and Lehtinen, M.: Ozone profile smoothness as a priori information in the inversion of limb measurements, Ann. Geophys., 22(10), 34113420, 2004.

Sofieva, V. F., Kyrölä, E., Hassinen, S., et al.: Global analysis of scintillation variance: Indication of gravity wave breaking in the polar winter upper stratosphere, Geophys. Res. Lett., 34, L03812, doi:10.1029/2006GL028132, 2007a.

Sofieva, V. F., Gurvich, A. S., Dalaudier, F., and Kan, V.: Reconstruction of internal gravity wave and turbulence parameters in the stratosphere using GOMOS scintillation measurements, J. Geophys. Res., 112, D12113, doi:10.1029/2006JD007483, 2007b.

Sofieva, V. F., Gurvich, A. S., and Dalaudier, F.: Gravity wave spectra parameters in 2003 retrieved from stellar scintillation measurements by GOMOS, Geophys. Res. Lett., 36, L05811, doi:10.1029/2008GL036726, 2009.

Tamminen, J., Kyrölä, E., and Sofieva, V.: Does a priori information improve occultation measurements?, in: Occultations for Probing Atmosphere and Climate, edited by: Kirchengast, G., Foelshe, U., and Steiner, A., Springer Verlag 2004, 87-98, 2004. 